\title{
Regularity of solutions to elliptic equations on Herz spaces with variable exponents
}

\section{Andrea Scapellato ${ }^{1 *}$ (1)}

\section{"Correspondence:}

scapellato@dmi.unict.it

'Dipartimento di Matematica e

Informatica, Università degli Studi di

Catania, Catania, Italy

\begin{abstract}
The aim of this paper is to show some regularity properties of solutions to $\mathcal{L} u=f$, where $\mathcal{L}$ is a second order nondivergence form operator and $f$ belongs to a homogeneous Herz space $\dot{K}_{q(\cdot)}^{\alpha, p(\cdot)}$ with two variable exponents.

MSC: Primary 42B37; secondary 35B65

Keywords: Herz spaces; Elliptic equations; VMO
\end{abstract}

\section{Introduction}

In this note we apply some recent estimates of singular integral operators and commutators to the study of regularity of solutions to second order elliptic equations with nondivergence principal part having discontinuous coefficients.

Let $\Omega \subset \mathbb{R}^{n}, n \geq 2$, be a bounded domain, and let us consider the partial differential equation

$$
\mathcal{L} u \equiv a_{i j}(x) D_{x_{i} x_{j}} u=f(x)
$$

where $a_{i j}, f: \Omega \rightarrow \mathbb{R}$. We require the ellipticity of the differential operator $\mathcal{L}$ imposing the positive definiteness of the coefficients matrix $\mathcal{A}(x)=\left\{a_{i j}(x)\right\}_{i, j=1, \ldots, n}$. In particular, $\mathcal{L}$ is strictly elliptic if

$$
\lambda(x)>\text { const }>0 \text { in } \Omega,
$$

where $\lambda(x)$ is the minimum eigenvalue of $\mathcal{A}(x)$. Also, the uniform ellipticity means boundedness of the spectrum of $\mathcal{A}(x)$,

$$
\frac{\Lambda(x)}{\lambda(x)} \leq \text { const in } \Omega
$$

where with $\Lambda(x)$ we denote the maximum eigenvalue of $\mathcal{A}(x)$.

As usual, we say that a function $u \in C^{2}(\Omega)$ is a classical solution to (1.1) if it satisfies the equation at each $x \in \Omega$. It is clear that in order to deduce the solvability to (1.1) in that

(c) The Author(s) 2019. This article is distributed under the terms of the Creative Commons Attribution 4.0 International License (http://creativecommons.org/licenses/by/4.0/), which permits unrestricted use, distribution, and reproduction in any medium, provided you give appropriate credit to the original author(s) and the source, provide a link to the Creative Commons license, and indicate if changes were made. 
rough sense, we should require some regularity assumptions on the coefficients $a_{i j}$ beside the strict and uniform ellipticity mentioned above.

In the framework of the Schauder theory of linear elliptic operators, we have results dealing with classical solvability and regularity for equation (1.1) with Hölder continuous coefficients $a_{i j} \in C^{0, \alpha}(\bar{\Omega}), \alpha \in(0,1)$, and $f \in C^{0, \alpha}(\bar{\Omega})$. However, it is well known that even the Poisson equation $\Delta u=f$ does not have a $C^{2}$-solution for continuous $f$.

This example justifies another definition of solution. Namely, it is common to define the concept of strong solution. We say that a strong solution to (1.1) is a twice weakly differentiable function, $u \in W^{2, p}(\Omega)$ for suitable $p \in(1, \infty)$, satisfying the equation $\mathcal{L} u=f$ almost everywhere (a.e.) in $\Omega$. That new concept of solution was meant also for $f \in L^{p}(\Omega)$ and $a_{i j} \in L^{\infty}(\Omega)$.

On the other hand, the only strict ellipticity and the fact that the coefficients $a_{i j}$ could be essentially bounded are not enough to deduce strong solvability of (1.1) as shown by Pucci. Precisely, from the studies of Pucci, it turns out that a satisfactory strong solvability theory of (1.1) can be built only with additional hypotheses on the coefficients $a_{i j}$ of $\mathcal{L}$. These assumptions should be either regularity hypotheses on the coefficients $a_{i j}$ or hypotheses on the spectrum of the matrix $\mathcal{A}(x)$ stronger than the strict and uniform ellipticity. For instance, if we assume that the coefficients $a_{i j}$ are continuous in $\bar{\Omega}$,

$$
a_{i j}(x) \in C^{0}(\bar{\Omega})
$$

the classical $L^{p}$-theory provides solvability and regularity for (1.1) in Sobolev spaces $W^{2, p}(\Omega)$ for any $p>1$.

For further details, see, for instance, the classical monographs of Ladyzhenskaya and Ural'tseva [1], Ladyzhenskaya, Solonnikov, and Ural'tseva [2], Miranda [3], and Gilbarg and Trudinger [4].

Over the years there was developed an analogue of the $L^{p}$-theory of elliptic (and parabolic) equations, weakening the continuity assumption on the coefficients of the principal part of the operators under consideration. Precisely, it is interesting to investigate the case of discontinuous coefficients. We recall the so-called Cordes condition, introduced by Cordes in the study of Hölder continuity of solutions to (1.1). This condition substitutes the continuity assumption with the requirement that all the eigenvalues $\lambda_{i}(x)$ of the matrix $\mathcal{A}(x)$ do not spread too much. The Cordes condition allowed Talenti to obtain strong solvability in $W^{2,2}(\Omega)$ of the Dirichlet problem for the operator $\mathcal{L}$, and starting from his paper, other authors studied other boundary value problems of elliptic and parabolic type.

Another class of discontinuous coefficients was introduced by Miranda, and it consists of all functions that belong to the Sobolev space $W^{1, n}(\Omega)$. In spite of the difference between these two types of discontinuity, the approaches in the study of boundary value problems are unified by the Miranda-Talenti inequality which allows us to obtain the constants that appear in the $L^{2}$-a priori estimates.

It is important to underline that all the assumptions above permit to derive regularity results in the frameworks of $W^{2,2}$ or $W^{2, p}$ with $p$ greater than, but sufficiently near to, 2 .

Finally, it is important to investigate the $L^{p}$-theory of elliptic and parabolic operators with principal coefficients $a_{i j}$ belonging to the Sarason class $V M O$ of functions having vanishing mean oscillation. In two pioneering scientific notes, Chiarenza, Frasca, and Longo modified the classical methods in order to obtain $L^{p}$ estimates for solutions to (1.1); the 
approach followed by Chiarenza, Frasca, and Longo is strictly related to the theory of Calderón and Zygmund and uses a representation formula for the second derivatives $D^{2} u$ of any solutions to (1.1) in terms of a singular integral that acts on $f$ and a commutator that acts on the same derivatives $D^{2} u$ and with density $a_{i j}(x)-a_{i j}(y)$. Thus, if the coefficients $a_{i j}$ have a vanishing mean oscillation, guaranteed by the assumption $a_{i j} \in V M O$, then the $L^{p}$-norm of $D^{2} u$ is bounded in terms of $L^{p}$-norm of $f$, and this result holds for any $p \in(1, \infty)$.

Taking into account that $V M O$ contains as proper subsets both $C^{0}(\bar{\Omega})$ and $W^{1, n}(\Omega)$, the $L^{p}$-theory of operators with $V M O$ principal coefficients generalized the theories known before 1990.

In this work the crucial assumption is $a_{i j} \in V M O$.

In the last years a lot of authors obtained regularity results in several spaces: for instance, we recall the regularity results obtained in the framework of Morrey spaces by Di Fazio and Ragusa in [5], generalized Morrey spaces by Scapellato in [6], and Herz spaces by Ragusa in [7] and Scapellato in [8]. In the first two papers, the authors use the representation formula for the second derivatives of a solution to the equations under consideration and apply the estimates contained in [9] and [10], respectively.

The regularity of solutions to differential problems was also extensively studied in the recent papers [11-13].

In this note, we obtain a regularity result in the framework of Herz spaces with variable exponents. The analysis of the boundedness of integral operators, the calculus of variations, the study of the regularity to solutions to partial differential equations and systems in such nonstandard functional spaces has shown a growing interest in recent years.

Our study starts from the boundedness results contained in [14] and uses the technique in [15] for interior estimates, based on the explicit representation formula for the second derivatives of a solution to (1.1).

In the next section we present some useful definitions and tools.

\section{Homogeneous Herz spaces with variable exponent}

Let $\Omega$ be a measurable set in $\mathbb{R}^{n}$. We firstly recall the definition of the Lebesgue spaces with variable exponent. For a deeper discussion of Lebesgue spaces with variable exponent, we refer the reader to [16].

Let $\mathcal{P}(\Omega)$ be the set of all Lebesgue measurable functions $p(\cdot): \Omega \rightarrow[1, \infty]$. The elements of $\mathcal{P}(\Omega)$ are called exponent functions. We denote by $\mathcal{B}(\Omega)$ the set of all functions $p(\cdot) \in \mathcal{P}(\Omega)$ such that the related Hardy-Littlewood maximal operator is bounded on $L^{p(\cdot)}(\Omega)$.

Throughout this paper we will set

$$
p_{-}=\operatorname{essinf}\{p(x): x \in \Omega\}, \quad p_{+}=\operatorname{esssup}\{p(x): x \in \Omega\} .
$$

Let $p(\cdot) \in \mathcal{P}(\Omega)$ and let $f$ be a Lebesgue measurable function. Let us define the modular functional associated with $p(\cdot)$ by

$$
\rho_{p(\cdot), \Omega}(f)=\int_{\Omega \backslash \Omega_{\infty}}|f(x)|^{p(x)} \mathrm{d} x+\|f\|_{L^{\infty}\left(\Omega_{\infty}\right)},
$$

where $\Omega_{\infty}=\{x \in \Omega: p(x)=\infty\}$. 
If $f$ is unbounded on $\Omega_{\infty}$ or if $f(\cdot)^{p(\cdot)} \notin L^{1}\left(\Omega \backslash \Omega_{\infty}\right)$, we define $\rho_{p(\cdot), \Omega}(f)=+\infty$. When $\left|\Omega_{\infty}\right|=0$, in particular when $p_{+}<\infty$, we let $\|f\|_{L^{\infty}\left(\Omega_{\infty}\right)}=0$. When $\left|\Omega \backslash \Omega_{\infty}\right|=0$, then $\rho_{p(\cdot), \Omega}(f)=\|f\|_{L^{\infty}\left(\Omega_{\infty}\right)}$. In situations where there is no ambiguity, we will simply write $\rho(f)$.

Definition 1 Let $\Omega$ be a measurable set in $\mathbb{R}^{n}$ and $p(\cdot) \in \mathcal{P}(\Omega)$. Let us define $L^{p(\cdot)}(\Omega)$ to be the set of Lebesgue measurable functions $f$ such that $\rho\left(\frac{f}{\lambda}\right)<\infty$ for some $\lambda>0$.

Also, for every compact set $K \subseteq \Omega$, the space $L_{\text {loc }}^{p(\cdot)}(\Omega)$ is defined by

$$
L^{p(\cdot)}(\Omega)=\left\{f \text { is measurable: } f \in L^{p(\cdot)}(K)\right\} .
$$

Definition 2 Let $p(\cdot), q(\cdot) \in \mathcal{P}(\Omega)$. The mixed Lebesgue sequence space with variable exponent $\ell^{q(\cdot)}\left(L^{p(\cdot)}\right)$ is the set of all sequences $\left\{f_{j}\right\}_{j \in \mathbb{N}}$ of measurable functions on $\mathbb{R}^{n}$ such that

$$
\left\|\left\{f_{j}\right\}_{j \in \mathbb{N}}\right\|_{\ell q(\cdot)\left(L^{p(\cdot)}\right)}=\inf \left\{\eta>0: Q_{\ell^{q(\cdot)}\left(L^{p \cdot \cdot)}\right)}\left(\left\{\frac{f_{j}}{\zeta}\right\}_{j \in \mathbb{N}}\right) \leq 1\right\}<\infty
$$

where

$$
Q_{\ell q(\cdot)\left(L^{p(\cdot))}\right.}\left(\left\{f_{j}\right\}_{j \in \mathbb{N}}\right)=\sum_{j=0}^{\infty} \inf \left\{\zeta_{j}>0: \int_{\mathbb{R}^{n}}\left(\frac{\left|f_{j}(x)\right|}{\zeta_{j}^{\frac{1}{q(x)}}}\right)^{p(x)} \mathrm{d} x \leq 1\right\} .
$$

We observe that, for $q_{+}<\infty$, we have that

$$
Q_{\ell q(\cdot)\left(L^{p(\cdot)}\right)}\left(\left\{f_{j}\right\}_{j \in \mathbb{N}}\right)=\sum_{j=0}^{\infty}\left\|\left|f_{j}\right|^{q(\cdot)}\right\|_{L^{\frac{p(\cdot)}{q(\cdot)}}} .
$$

Let $B_{k}=\left\{x \in \mathbb{R}^{n}:|x| \leq 2^{k}\right\}, C_{k}=B_{k} \backslash B_{k-1}, \chi_{k}:=\chi_{C_{k}}, k \in \mathbb{Z}$.

Definition 3 Let $\alpha \in \mathbb{R}^{n}, q(\cdot), p(\cdot) \in \mathcal{P}\left(\mathbb{R}^{n}\right)$. The homogeneous Herz space with variable exponent $\dot{K}_{p(\cdot)}^{\alpha, q(\cdot)}$ is defined as follows:

$$
\dot{K}_{p(\cdot)}^{\alpha, q(\cdot)}=\left\{f \in L_{\mathrm{loc}}^{p(\cdot)}\left(\mathbb{R}^{n} \backslash\{0\}\right):\|f\|_{\left.\dot{K}_{p(\cdot)}^{\alpha, q(\cdot)}<\infty\right\},}\right.
$$

where

$$
\begin{aligned}
\|f\|_{\dot{K}_{p(\cdot)}^{\alpha, q(\cdot)}} & =\left\|\left\{2^{k \alpha}\left|f \chi_{k}\right|\right\}_{k \in \mathbb{N}}\right\|_{\ell q(\cdot)\left(L^{p \cdot(\cdot)}\right)} \\
& =\inf \left\{\eta>0: \sum_{k=-\infty}^{\infty}\left\|\left(\frac{2^{k \alpha}\left|f \chi_{k}\right|}{\eta}\right)^{q(\cdot)}\right\|_{L^{\frac{p(\cdot)}{q(\cdot)}}} \leq 1\right\} .
\end{aligned}
$$

\section{Calderón-Zygmund operators, BMO and VMO spaces}

In this section we review some of the standard facts on Calderón-Zygmund operators and their commutators. With $\mathscr{S}\left(\mathbb{R}^{n}\right)$ we denote the Schwartz class and with $\mathscr{S}^{\prime}\left(\mathbb{R}^{n}\right)$ we indicate the space of tempered distributions. Let us start with the following definition (see $[17,18])$. 
Definition 4 Let $T$ be a bounded linear operator from $\mathscr{S}\left(\mathbb{R}^{n}\right)$ to $\mathscr{S}^{\prime}\left(\mathbb{R}^{n}\right)$. We say that $T$ is a standard operator if it satisfies the following conditions:

- $T$ extends to a bounded linear operator on $L^{2}\left(\mathbb{R}^{n}\right)$;

- There exists a function $K(x, y)$ defined on $\left\{(x, y) \in \mathbb{R}^{n} \times \mathbb{R}^{n}: x \neq y\right\}$ such that

$$
|K(x, y)| \leq \frac{C}{|x-y|^{n}}
$$

where $C>0$;

- $\langle T f, g\rangle=\int_{\mathbb{R}^{n}} \int_{\mathbb{R}^{n}} K(x, y) f(y) g(x) \mathrm{d} x \mathrm{~d} y$ for $f, g \in \mathscr{S}\left(\mathbb{R}^{n}\right)$ such that $\operatorname{supp}(f) \cap \operatorname{supp}(g)=\varnothing$.

A standard operator $T$ is called a $\gamma$-Calderón-Zygmund operator if $K$ is a standard kernel satisfying

$$
\begin{aligned}
& |K(x, y)-K(z, y)| \leq C \frac{|x-z|^{\gamma}}{|x-y|^{n+\gamma}}, \\
& |K(y, x)-K(y, z)| \leq C \frac{|x-z|^{\gamma}}{|x-y|^{n+\gamma}},
\end{aligned}
$$

if $|x-z|<\frac{1}{2}|x-y|$ for some $\left.\left.\gamma \in\right] 0,1\right]$.

The commutator of the Calderón-Zygmund operator is defined by

$$
[b, T] f(x)=b(x) T f(x)-T(b f)(x) .
$$

In 1983, Journé proved that a $\gamma$-Calderón-Zygmund operator is bounded on $L^{p}\left(\mathbb{R}^{n}\right)$ (see [19]). Coifman, Rochberg, and Weiss in [20] proved that the commutator $[b, T]$ is bounded on $L^{p}\left(\mathbb{R}^{n}\right)$ for $\left.p \in\right] 0,1[$.

Kovácik and Rákosník in [21] introduced Lebesgue spaces and Sobolev spaces with variable exponent. For a recent treatment of Lebesgue spaces with variable exponent, we refer the reader to [16].

In the last decades, there was an increasing interest in the study of functional spaces having variable exponent thanks to the wide variety of applications, for instance, in fluid dynamics and differential equations.

In particular, Herz spaces play an important role in harmonic analysis. In this paper, we will apply the theory of regularity of solutions to partial differential equations to the main results contained in [14]. They deal with the boundedness of Calderón-Zygmund operator and their commutator on Herz spaces with two variable exponents $p(\cdot), q(\cdot)$.

In order to develop a satisfactory theory of regularity of solutions to linear elliptic differential equations, following the pioneering scientific note [15], we will assume that the coefficients of the differential operators under consideration belong to the Sarason class of functions having vanishing mean oscillation. This requirement on the coefficients allows us to consider also the case that these coefficients are discontinuous.

First of all we recall the definition of $B M O$ space due to John and Nirenberg (see [22]).

Definition 5 We define the space $B M O\left(\mathbb{R}^{n}\right)$ of functions having bounded mean oscillation as

$$
\operatorname{BMO}\left(\mathbb{R}^{n}\right)=\left\{b \in L_{\mathrm{loc}}^{1}\left(\mathbb{R}^{n}\right):\|b\|_{*}<\infty\right\},
$$


where

$$
\|b\|_{*}=\sup _{B \subseteq \mathbb{R}^{n}} \frac{1}{|B|} \int_{B}\left|b(x)-b_{B}\right| \mathrm{d} x,
$$

where $B$ is a generic ball in $\mathbb{R}^{n}$ and $b_{B}$ stands for the integral average of the function $b$ over the sphere $B$.

In the next definition we define a proper subset of the space $B M O$ studied by Sarason (see [23]).

Definition 6 We define the space $V M O\left(\mathbb{R}^{n}\right)$ of functions having vanishing mean oscillation as

$$
\operatorname{VMO}\left(\mathbb{R}^{n}\right)=\left\{b \in B M O\left(\mathbb{R}^{n}\right): \lim _{r \rightarrow 0^{+}} \gamma_{b}(r)=0\right\},
$$

where

$$
\gamma_{b}(r)=\sup _{\rho \leq r} \frac{1}{\left|B_{\rho}\right|} \int_{B_{\rho}}\left|b(x)-b_{B_{\rho}}\right| \mathrm{d} x
$$

and $B_{\rho}$ varies in the class of balls in $\mathbb{R}^{n}$ having radius $\rho$. We call $\gamma_{b}$ the $V M O$-modulus of the function $b$.

In a similar way, we can define the spaces $B M O(\Omega)$ and $V M O(\Omega)$ of functions defined on a domain $\Omega \subset \mathbb{R}^{n}$, replacing $B$ and $B_{\rho}$ by the intersections of the respective balls with $\Omega$.

It is worth pointing out that applying the classical Poincaré inequality, it follows that $W^{1, n}\left(\mathbb{R}^{n}\right) \subset V M O$ and, further on, $W^{\theta, n / \theta}\left(\mathbb{R}^{n}\right) \subset V M O$ for $0<\theta<1$ as shows the function $f_{\alpha}(x)=|\log | x||^{\alpha}$ for $0<\alpha<1$. Straightforward calculations yield that $f_{\alpha} \in V M O$ for every $\alpha \in(0,1), f_{\alpha} \in W^{1, n}$ for $\alpha \in\left(0,1-\frac{1}{n}\right)$, while $f_{\alpha} \notin W^{1, n}$ for $\alpha \in\left[1-\frac{1}{n}, 1\right)$.

\section{Main result}

In the sequel, let $n \geq 3$ and

$$
L:=\sum_{i, j=1}^{n} a_{i j}(x) \partial_{x_{i} x_{j}}
$$

where

(1) $a_{i j} \in V M O \cap L^{\infty}(\Omega)$ for all $i, j=1, \ldots, n$ and a.a. $x \in \Omega$;

(2) $a_{i j}(x)=a_{j i}(x)$ for all $i, j=1, \ldots, n$ and a.a. $x \in \Omega$;

(3) $\exists v>0: v^{-1}|\xi|^{2} \leq \sum_{i, j=1}^{n} a_{i j}(x) \xi_{i} \xi_{j} \leq v|\xi|^{2}$, a.e. in $B$, for all $\xi \in \mathbb{R}^{n}$.

In the following we set

$$
\Gamma(x, t)=\frac{1}{(n-2) \omega_{n}\left(\operatorname{det} a_{i j}(x)\right)^{12}}\left(\sum_{i, j=1}^{n} A_{i j}(x) t_{i} t_{j}\right)^{\frac{2-n}{2}}
$$

for a.a. $x \in B$, for $t \in \mathbb{R}^{n} \backslash\{0\}$, where we denote by $A_{i j}(x)$ the entries of the inverse of the matrix $\left(a_{i j}(x)\right)_{i, j=1, \ldots, n}$. Let us observe that if $\tilde{B}$ is a subset of $B$ in which conditions (1) and 
(2) hold everywhere, for fixed $x_{0} \in \tilde{B}, \Gamma\left(x_{0}, t\right)$ is a fundamental solution for the operator

$$
L_{0} u(x)=\sum_{i, j=1}^{n} a_{i j}\left(x_{0}\right) u_{x_{i} x_{j}}(x)
$$

Also, we set

$$
\Gamma_{i}(x, t)=\frac{\partial}{\partial t_{i}} \Gamma(x, t), \quad \Gamma_{i j}(x, t)=\frac{\partial^{2}}{\partial t_{i} \partial t_{j}} \Gamma(x, t) .
$$

Furthermore, we set

$$
M:=\max _{i, j=1, \ldots, n n|\alpha| \leq 2 n} \max _{|x|}\left\|\frac{\partial^{\alpha} \Gamma_{i j}(x, t)}{\partial t^{\alpha}}\right\|_{L^{\infty}(\Omega \times \Sigma)} .
$$

It is well known that $\Gamma_{i j}(x, t)$ are Calderón-Zygmund kernels in the $t$ variable.

Let us start with some useful tools. For the proofs, we refer the reader to [14].

Lemma 7 Let $p_{h} \in \mathcal{B}\left(\mathbb{R}^{n}\right)$ for $h=1,2$, then there exist constants $0<t_{h 1}, t_{h 2}<1$, and $C>0$ such that, for all balls $B \subset \mathbb{R}^{n}$ and all measurable subsets $R \subset B$,

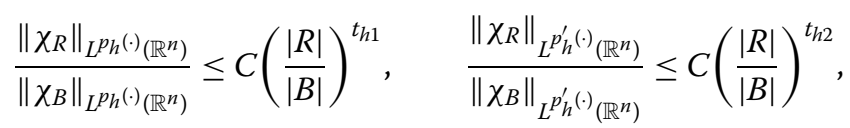

where with $p_{h}^{\prime}(\cdot)$ is the conjugate exponent function defined as

$$
\frac{1}{p_{h}(x)}+\frac{1}{p_{h}^{\prime}(x)}=1, \quad x \in \mathbb{R}^{n} .
$$

Theorem 8 Suppose that $p_{1} \in \mathcal{B}\left(\mathbb{R}^{n}\right), q_{1}(\cdot), q_{2}(\cdot) \in \mathcal{P}\left(\mathbb{R}^{n}\right)$ with $\left(q_{2}\right)_{-} \geq\left(q_{1}\right)_{+}$. If $-n t_{12}<$ $\alpha<n t_{11}$, with $t_{11}, t_{12}$ as in Lemma 7 , then the operator $T$ of Definition 4 is bounded from $\dot{K}_{p_{1}(\cdot)}^{\alpha, q_{2}(\cdot)}\left(\mathbb{R}^{n}\right)$ to $\dot{K}_{p_{1}(\cdot)}^{\alpha, q_{1}(\cdot)}\left(\mathbb{R}^{n}\right)$.

Theorem 9 Let $b \in B M O\left(\mathbb{R}^{n}\right)$. Suppose that $p_{1}(\cdot) \in \mathcal{B}\left(\mathbb{R}^{n}\right), q_{1}(\cdot), q_{2}(\cdot) \in \mathcal{P}\left(\mathbb{R}^{n}\right)$ with $\left(q_{2}\right)_{-} \geq$ $\left(q_{1}\right)_{+}$. If $-n t_{12}<\alpha<n t_{11}$, with $t_{11}, t_{12}$ as in Lemma 7 , then the commutator $[b, T]$ is bounded from $\dot{K}_{p_{1}(\cdot)}^{\alpha, q_{2}(\cdot)}\left(\mathbb{R}^{n}\right)$ to $\dot{K}_{p_{1}(\cdot)}^{\alpha, q_{1}(\cdot)}\left(\mathbb{R}^{n}\right)$.

Now we are ready to show our main result. The proof is based on the representation formula for the second derivatives of a solution in the class $W_{0}^{2, p}$.

Theorem 10 Under assumptions (1), (2), (3), $p \in] 1, \infty\left[, p_{1}(\cdot), q(\cdot)=q_{1}(\cdot)=q_{2}(\cdot)\right.$, and $\alpha$ as in Theorems 8 and 9, there exist positive numbers $C=C\left(n, p, p_{1}, q, M, \alpha\right)$ and $\rho_{0}=\rho_{0}(C, n)$ such that, for any ball $B_{r} \Subset \Omega$ with $r<\rho_{0}$ and any $u \in W_{0}^{2, p}\left(B_{R}\right)$ such that $u_{x_{i} x_{j}} \in \dot{K}_{p_{1}(\cdot)}^{\alpha, q(\cdot)}\left(B_{r}\right)$ and $\mathcal{L} u=f(x)$, with $f \in \dot{K}_{p_{1}(\cdot)}^{\alpha, q(\cdot)}\left(B_{r}\right)$, we have

$$
\left\|u_{x_{i} x_{j}}\right\|_{\dot{K}_{p_{1}(\cdot)}^{\alpha, q(\cdot)}\left(B_{r}\right)} \leq C\|f\|_{\dot{K}_{p_{1}(\cdot)}^{\alpha, q(\cdot)}\left(B_{r}\right)}, \quad \forall i, j=1, \ldots, n .
$$


Proof Let $n \geq 3, B$ be an open ball in $\mathbb{R}^{n},\left(a_{i j}\right)_{i, j=1, \ldots, n}$ be as above and $u \in W_{0}^{2, p}(B)$. Then, for a.a. $x \in B$, the following representation formula holds (see [15]):

$$
\begin{aligned}
u_{x_{i} x_{j}}(x)= & \text { P.V. } \int_{B} \Gamma_{i j}(x, x-y) \cdot\left[\sum_{h, k=1}^{n}\left(a_{h k}(x)-a_{h k}(y)\right) u_{x_{h} x_{k}}(y)+L u(y)\right] \mathrm{d} y \\
& +L u(x) \int_{|t|=1} \Gamma_{i}(x, t) t_{j} \mathrm{~d} \sigma_{t} .
\end{aligned}
$$

From Theorem 8 , it follows that, for any $h \in \dot{K}_{p_{1}(\cdot)}^{\alpha, q_{1}(\cdot)}\left(\mathbb{R}^{n}\right)$, the following inequality holds:

$$
\|T(h)\|_{\dot{K}_{p_{1}(\cdot)}^{\alpha, q_{2}(\cdot)}\left(\mathbb{R}^{n}\right)} \leq C\|h\|_{\dot{K}_{p_{1}(\cdot)}^{\alpha, q_{1}(\cdot)}\left(\mathbb{R}^{n}\right)} .
$$

Furthermore, from Theorem 9, it follows that, for any $h \in \dot{K}_{p_{1}(\cdot)}^{\alpha, q_{1}(\cdot)}\left(\mathbb{R}^{n}\right)$ and $b \in B M O\left(\mathbb{R}^{n}\right)$, we have

$$
\|[b, T](h)\|_{\dot{K}_{p_{1}(\cdot)}^{\alpha, q_{2}(\cdot)}\left(\mathbb{R}^{n}\right)} \leq C\|b\|_{*}\|h\|_{\dot{K}_{p_{1}(\cdot)}^{\alpha, q_{1}(\cdot)}\left(\mathbb{R}^{n}\right)} .
$$

Using the representation formula (4.1) and estimates (4.2), (4.3), we obtain the desired estimate.

\section{Acknowledgements}

The author is grateful to the anonymous referees for their valuable suggestions and comments.

\section{Funding}

Not applicable.

\section{Availability of data and materials}

Data sharing not applicable to this article as no datasets were generated or analysed during the current study.

\section{Competing interests}

The author declares that he has no competing interests regarding the publication of this paper.

\section{Authors' contributions}

The author read and approved the final manuscript.

\section{Publisher's Note}

Springer Nature remains neutral with regard to jurisdictional claims in published maps and institutional affiliations.

Received: 30 August 2018 Accepted: 25 December 2018 Published online: 05 January 2019

\section{References}

1. Ladyzhenskaya, O.A., Ural'tseva, N.N.: Linear and Quasilinear Elliptic Equations. Mathematics in Science and Engineering, vol. 46. Academic Press, New York (1968)

2. Ladyzhenskaya, O.A., Solonnikov, V.A., Ural'tseva, N.N.: Linear and Quasilinear Equations of Parabolic Type. Transl. Math. Monographs, vol. 23. Am. Math. Soc., Providence (1968)

3. Miranda, C.: Partial Differential Equations of Elliptic Type, 2nd edn. Springer, Berlin (1970)

4. Gilbarg, D., Trudinger, N.S.: Elliptic Partial Differential Equations of Second Order, 3rd edn. Springer, Berlin (1997)

5. Di Fazio, G., Ragusa, M.A.: Interior estimates in Morrey spaces for strong solutions to nondivergence form equations with discontinuous coefficients. J. Funct. Anal. 112, 241-256 (1993)

6. Scapellato, A.: On some qualitative results for the solution to a Dirichlet problem in local generalized Morrey spaces. AIP Conf. Proc. 1798, 020138 (2017). https://doi.org/10.1063/1.4972730

7. Ragusa, M.A.: Homogeneous Herz spaces and regularity results. Nonlinear Anal., Theory Methods Appl. 71(12), e1909-e1914 (2009)

8. Scapellato, A.: Homogeneous Herz spaces with variable exponents and regularity results. Electron. J. Qual. Theory Differ. Equ. 2018, 82 (2018). https://doi.org/10.14232/ejqtde.2018.1.82

9. Di Fazio, G., Ragusa, M.A.: Commutators and Morrey spaces. Boll. Unione Mat. Ital. 5(3), 323-332 (1991)

10. Scapellato, A.: Some properties of integral operators on generalized Morrey spaces. AlP Conf. Proc. 1863, 510004 (2017). https://doi.org/10.1063/1.4992662 
11. Musaev, H.K., Shakhmurov, V.B.: Regularity properties of degenerate convolution-elliptic equations. Bound. Value Probl. 2016, 50 (2016)

12. Shakhmurov, V.B.: Nonlocal problems for Boussinesq equations. Nonlinear Anal., Theory Methods Appl. 142, 134-151 (2016)

13. Musaev, H.K., Shakhmurov, V.B.: B-coercive convolution equations in weighted function spaces and their applications. Ukr. Math. J. 69(10), 1607-1630 (2018)

14. Abdalrhman, O., Abdalmonem, A., Tao, S.P.: Boundedness of Calderón-Zygmund operator and their commutator on Herz spaces with variable exponent. Appl. Math. 8, 428-443 (2017). https://doi.org/10.4236/am.2017.84035

15. Chiarenza, F., Frasca, M., Longo, P.: Interior $W^{2, P}$ estimates for non-divergence elliptic equations with discontinuous coefficients. Ric. Mat. 40, 149-168 (1991)

16. Cruz-Uribe, D.V., Fiorenza, A.: Variable Lebesgue Spaces: Foundations and Harmonic Analysis. Applied and Numerical Harmonic Analysis. Springer, New York (2013)

17. Calderón, A., Zygmund, A.: On singular integrals. Am. J. Math. 78, 289-309 (1956). https://doi.org/10.2307/2372517

18. Calderón, A., Zygmund, A.: On singular integral with variable kernels. Appl. Anal. 7, 221-238 (1978). https://doi.org/10.1080/00036817808839193

19. Journé, J.-L.: Calderón-Zygmund Operators, Pseudo-Differential Operators and the Cauchy Integral of Calderón. Lecture Notes in Math., vol. 994. Springer, Berlin (1983)

20. Coifman, R., Rochberg, R., Weiss, G.: Factorization theorems for Hardy spaces in several variables. Ann. Math. 103, 611-635 (1976). https://doi.org/10.2307/1970954

21. Kováčik, O., Rákosník, J.: On spaces $L^{p(x)}$ and $W^{k, p(x)}$. Czechoslov. Math. J. 41, 592-618 (1991)

22. John, F., Nirenberg, L.: On functions of bounded mean oscillation. Commun. Pure Appl. Math. 14, 415-426 (1961)

23. Sarason, D.: On functions of vanishing mean oscillation. Transl. Am. Math. Soc. 207, 391-405 (1975)

\section{Submit your manuscript to a SpringerOpen ${ }^{\circ}$ journal and benefit from:}

- Convenient online submission

- Rigorous peer review

- Open access: articles freely available online

- High visibility within the field

- Retaining the copyright to your article

Submit your next manuscript at $\gg$ springeropen.com 